\title{
HOW TO BURN A GRAPH
}

\author{
Anthony Bonato, ${ }^{1}$ Jeannette Janssen, ${ }^{2}$ and Elham Roshanbin ${ }^{2}$ \\ ${ }^{I}$ Department of Mathematics, Ryerson University, Toronto, Ontario, Canada \\ ${ }^{2}$ Department of Mathematics and Statistics, Dalhousie University, Halifax, Nova \\ Scotia, Canada
}

Abstract We introduce a new graph parameter called the burning number, inspired by contact processes on graphs such as graph bootstrap percolation, and graph searching paradigms such as Firefighter. The burning number measures the speed of the spread of contagion in a graph; the lower the burning number, the faster the contagion spreads. We provide a number of properties of the burning number, including characterizations and bounds. The burning number is computed for several graph classes, and is derived for the graphs generated by the Iterated Local Transitivity model for social networks.

\section{INTRODUCTION}

The spread of social influence is an active topic in social network analysis; see, for example [4, 9, 13, 14, 17, 19]. A recent study on the spread of emotional contagion in Facebook [16] has highlighted the fact that the underlying network is an essential factor; in particular, in-person interaction and nonverbal cues are not necessary for the spread of the contagion. Hence, agents in the network spread the contagion to their friends or followers, and the contagion propagates over time. If the goal was to minimize the time it took for the contagion to reach the entire network, then which agents would you target with the contagion, and in which order?

As a simplified, deterministic approach to these questions, we consider a new approach involving a graph process that we call burning. Burning is inspired by graph theoretic processes such as Firefighting [5, 8, 10, 11], graph cleaning [1], and graph bootstrap percolation [3]. There are discrete time-steps or rounds. Each node is either burned or unburned; if a node is burned, then it remains in that state until the end of the process. In every round, we choose one additional unburned node to burn (if such a node is available). Once a node is burned in round $t$, in round $t+1$, each of its unburned neighbors becomes burned. The process ends when all nodes are burned. The burning number of a graph $G$, written by $b(G)$, is the minimum number of rounds needed for the process to end. For example, it is straightforward to see that $b\left(K_{n}\right)=2$. However, even for a relatively simple graph

A preliminary version of this work was presented at the 11th Workshop on Algorithms and Models for the Web Graph (WAW'14), Beijing, China, December 2014.

Address correspondence to Anthony Bonato, Ryerson University, 350 Victoria St West, Toronto, ON, M5B 2K3 Canada. Email: abonato@ryerson.ca

Color versions of one or more of the figures in the article can be found online at www.tandfonline.com/uinm. 


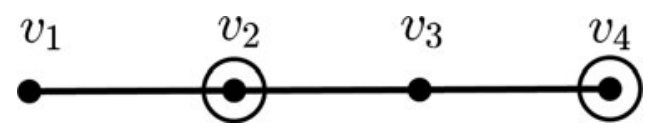

Figure 1 Burning the path $P_{4}$ (the open circles represent burned nodes).

such as the path $P_{n}$ on $n$ nodes, computing the burning number is more complex; in fact, $b\left(P_{n}\right)=\left\lceil n^{1 / 2}\right\rceil$ as stated further on, in Theorem 2.9.

Burning may be viewed as a simplified model for the spread of social contagion in a social network such as Facebook or Twitter. The lower the value of $b(G)$, the faster it is to spread such contagion in the graph $G$. Suppose that in the process of burning a graph $G$, we eventually burned the whole graph $G$ in $k$ steps, and for each $i, 1 \leq i \leq k$, we denote the node that we burn in the $i$ th step by $x_{i}$. We call such a node simply a source of fire. The sequence $\left(x_{1}, x_{2}, \ldots, x_{k}\right)$ is called a burning sequence for $G$. With this notation, the burning number of $G$ is the length of a shortest burning sequence for $G$; such a burning sequence is referred to as optimal. For example, for the path $P_{4}$ with node set $\left\{v_{1}, v_{2}, v_{3}, v_{4}\right\}$, the sequence $\left(v_{2}, v_{4}\right)$ is an optimal burning sequence; see Figure 1. Note that for a graph $G$ with at least two nodes, we have that $b(G) \geq 2$.

The goal of the current article is to introduce the burning number and explore its core properties. A characterization of burning number via a decomposition into trees is given in Theorem 2.4. As proven in [7], computing the burning number of a graph is NP-complete, even for trees of maximum degree three, spider graphs, and disconnected graphs such as path-forests. As such, we provide bounds on the burning number in terms of spanning trees, which are useful in many cases when computing the burning number. See Lemma 2.11 for bounds on the burning number. We compute the burning number on the Iterated Local Transitivity model for social networks (introduced in [6]); see Theorem 4.1. In the final section, we summarize our results and present open problems for future work.

\section{PROPERTIES OF THE BURNING NUMBER}

In this section, we collect a number of results on the burning number, ranging from characterizations, bounds, to computing the burning number on certain kinds of graphs. We first need some terminology. For further background, see [21]. If $G$ is a graph and $v$ is a node of $G$, then the eccentricity of $v$ is defined as $\max \{d(v, u): u \in V(G)\}$. The radius of $G$ is the minimum eccentricity over the set of all nodes in $G$. The center of $G$ consists of the nodes in $G$ with minimum eccentricity. Given a positive integer $k$, the $k$-th closed neighborhood of $v$ is defined to be the set $\{u \in V(G): d(u, v) \leq k\}$ and is denoted by $N_{k}[v]$; we denote $N_{1}[v]$ simply by $N[v]$. We sometimes use the notation $N_{k}^{G}[v]$ to emphasize that we consider the $k$-th closed neighbourhood of node $v$ in a specified graph $G$.

Suppose that $\left(x_{1}, x_{2}, \ldots, x_{k}\right)$ is a burning sequence for a given graph $G$. For $1 \leq i \leq$ $k$, the fire started at $x_{i}$ will burn only all the nodes within distance $k-i$ from $x_{i}$ by the end of the $k$-th step. On the other hand, every node $v \in V(G)$ must be either a source of fire, or burned from at least one of the sources of fire by the end of the $k$-th step. In other words, any node of $G$ must be an element of $N_{k-i}\left[x_{i}\right]$, for some $1 \leq i \leq k$. Moreover, for each pair $i$ and $j$, with $1 \leq i<j \leq k$, we must have $d\left(x_{i}, x_{j}\right) \geq j-i$. Since, otherwise, if $d\left(x_{i}, x_{j}\right)=l<j-i$, then $x_{j}$ will be burned at stage $l+i(<j)$, which is a contradiction. Therefore, we can see that $\left(x_{1}, x_{2}, \ldots, x_{k}\right)$ forms a burning sequence for $G$ if and only if, 
for each pair $i$ and $j$, with $1 \leq i<j \leq k, d\left(x_{i}, x_{j}\right) \geq j-i$, and the following set equation holds:

$$
N_{k-1}\left[x_{1}\right] \cup N_{k-2}\left[x_{2}\right] \cup \ldots \cup N_{0}\left[x_{k}\right]=V(G) .
$$

A covering of $G$ is a set of subsets of the nodes of $G$ whose union is $V(G)$. The above observation, shows that the burning problem is basically a covering problem using closed neighborhoods with a restriction on their radius. Hence, it seems that by finding a covering for a graph $G$ using a limited number of connected subgraphs with restricted radius, we may find a bound on the burning number of $G$, as the following theorem shows.

Theorem 2.1. If, in a graph $G$, there exists a collection of connected subgraphs $\left\{C_{1}, C_{2}, \ldots, C_{t}\right\}$, each of radius at most $k$, which cover all the nodes of $G$, then $b(G) \leq t+k$.

Proof. We define a burning sequence $\left(x_{1}, x_{2}, \ldots, x_{t^{\prime}+k^{\prime}}\right)$, with $t^{\prime} \leq t$ and $k^{\prime} \leq k$ for $G$, as follows. Let $x_{1}$ be a center of the induced subgraph $G\left[C_{1}\right]$. Then, for $i \geq 2$, we let $x_{i}$ be a central node in $C_{j}$, with $j \geq i$, if none of the central nodes of $C_{j}$ are burned before the $i$ th step, where $j$ is the smallest index that satisfies this condition. We continue to choose $x_{i}$ 's by this rule until at some step $t^{\prime} \leq t$, by burning $x_{1}, x_{2}, \ldots, x_{t^{\prime}}$, each $C_{i}, 1 \leq i \leq t$ contains a burned center.

Now, for $j \geq 1$, we choose $x_{t^{\prime}+j}$ to be a node in $G$ that is not burned before the $\left(t^{\prime}+j\right)$ th step. Because the radius of each $C_{i}$ is at most $k$, then for some $k^{\prime} \leq k$, after $k^{\prime}$ more steps, every node in $G$ must be burned. Thus, $b(G) \leq t^{\prime}+k^{\prime} \leq t+k$.

We present another bound for the burning number of a graph using coverings. The proof is analogous to the one of Theorem 2.1, and so is omitted.

Theorem 2.2. If $\left\{C_{1}, C_{2}, \ldots, C_{t}\right\}$ is a covering of the nodes of a graph $G$, where each $C_{i}$ is a connected subgraph of radius at most $k-i$, and $t \leq k$, then $b(G) \leq k$.

We have the following immediate corollary.

Corollary 2.3. If $\left(x_{1}, x_{2}, \ldots, x_{k}\right)$ is a sequence of nodes in a graph $G$, such that $N_{k-1}\left[x_{1}\right] \cup$ $N_{k-2}\left[x_{2}\right] \cup \ldots \cup N_{0}\left[x_{k}\right]=V(G)$, then $b(G) \leq k$.

Proof. Set $C_{i}=N_{k-i}\left[x_{i}\right]$, for $1 \leq i \leq k$, and apply Theorem 2.2.

We consider the burning problem for connected graphs. Note that, as is the case for many graph parameters, the burning number of a disconnected graph $G$ with components $G_{1}, G_{2}, \ldots, G_{t}$, where $t \geq 2$, does not necessarily satisfy the equality $b(G)=b\left(G_{1}\right)+$ $b\left(G_{2}\right)+\cdots+b\left(G_{t}\right)$. For example, the disjoint union of $t$ paths of order 2 has burning number $t+1$.

The following theorem provides an alternative characterization of the burning number. The depth of a node in a rooted tree is the number of edges in a shortest path from the node to the tree's root. The height of a rooted tree $T$ is the greatest depth in $T$. A rooted tree partition of $G$ is a collection of rooted trees that are subgraphs of $G$, with the property that the node sets of the trees partition $V(G)$. 
Theorem 2.4. Burning a graph $G$ in $k$ steps is equivalent to finding a rooted tree partition into $k$ trees $T_{1}, T_{2}, \ldots, T_{k}$, with heights at most $(k-1),(k-2), \ldots, 0$, respectively, such that for every $1 \leq i, j \leq k$, the distance between the roots of $T_{i}$ and $T_{j}$ is at least $|i-j|$.

Proof. Assume that $\left(x_{1}, x_{2}, \ldots, x_{k}\right)$ is a burning sequence for $G$. For all $1 \leq i \leq k$, after $x_{i}$ is burned, in each round $t>i$ those unburned nodes of $G$ in the $(t-i)$-neighborhood of $x_{i}$ will burn. Hence, any node $v$ is burned by receiving fire via a shortest path of burned nodes from a fire source such as $x_{i}$ (this path can be of length zero in the case that $\left.v=x_{i}\right)$. Hence, we may define a surjective function $f: V(G) \rightarrow\left\{x_{1}, x_{2}, \ldots, x_{k}\right\}$, with $f(v)=x_{i}$ if $v$ receives fire from $x_{i}$, where $i$ is chosen with the smallest index. Now $\left\{f^{-1}\left(x_{1}\right), f^{-1}\left(x_{2}\right), \ldots, f^{-1}\left(x_{k}\right)\right\}$ forms a partition of $V(G)$ such that $G\left[f^{-1}\left(x_{i}\right)\right]$ (that is, the subgraph induced by $\left.f^{-1}\left(x_{i}\right)\right)$ forms a connected subgraph of $G$. Since every node $v$ in $f^{-1}\left(x_{i}\right)$ receives the fire spread from $x_{i}$ through a shortest path between $x_{i}$ and $v$, by deleting extra edges in $G\left[f^{-1}\left(x_{i}\right)\right]$ we can make a rooted subtree of $G$, called $T_{i}$ with root $x_{i}$. Because every node is burned after $k$ steps, the distance between each node on $T_{i}$ and $x_{i}$ is at most $k-i$. Therefore, the height of $T_{i}$ is at most $k-i$.

Conversely, suppose that we have a decomposition of the nodes of $G$ into $k$ rooted subtrees $T_{1}, T_{2}, \ldots, T_{k}$, such that for each $1 \leq i \leq k, T_{i}$ is of height at most $k-i$. Assume that $x_{1}, x_{2}, \ldots, x_{k}$ are the roots of $T_{1}, T_{2}, \ldots, T_{k}$, respectively, and for each pair $i$ and $j$, with $1 \leq i<j \leq k, d\left(x_{i}, x_{j}\right) \geq j-i$. Then $\left(x_{1}, x_{2}, \ldots, x_{k}\right)$ is a burning sequence for $G$, because the distance between any node in $T_{i}$ and $x_{i}$ is at most $k-i$. Thus, after $k$ steps, the graph $G$ will be burned.

Figure 2 illustrates Theorem 2.4. The burning sequence is $\left(x_{1}, x_{2}, x_{3}\right)$. We have shown the decomposition of $G$ into subgraphs $T_{1}, T_{2}$, and $T_{3}$ based on this burning sequence by drawing dashed curves around the corresponding subgraphs. Each node has been indexed by a number corresponding to the step that it is burned.

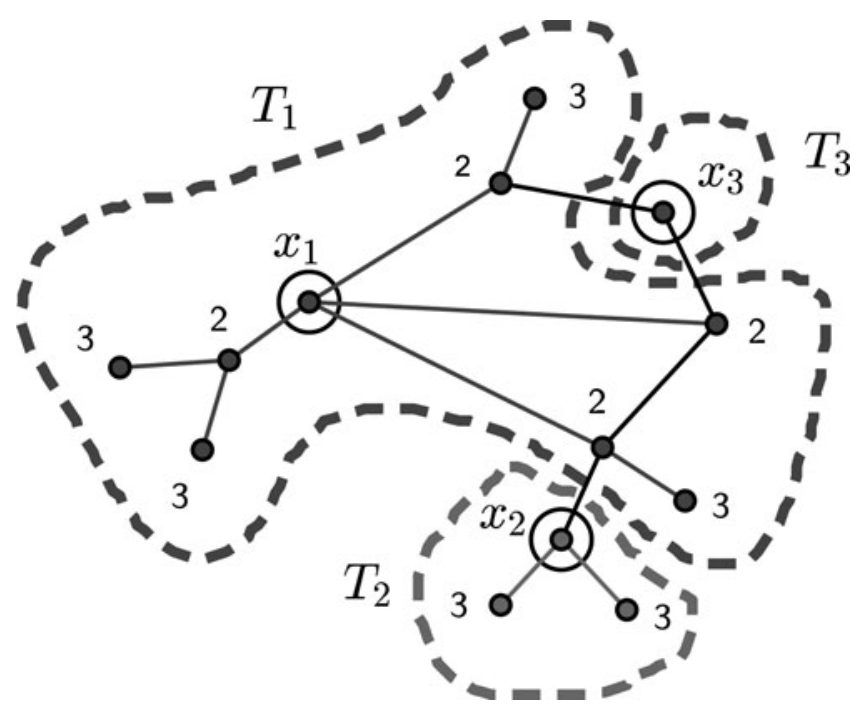

Figure 2 A rooted tree partition. 
The following corollary is useful for determining the burning number of a graph, because it reduces the problem of burning a graph to burning its spanning trees. Note that for a spanning subgraph $H$ of $G$, it is evident that $b(G) \leq b(H)$. This follows because, by (2.1), every optimal burning sequence for $H$ induces a node covering for $V(G)=V(H)$, and therefore, by Corollary 2.3, induces a burning sequence of, at most, the same length for $G$.

Corollary 2.5. For a graph $G$ we have that

$$
b(G)=\min \{b(T): T \text { is a spanning subtree of } G\} .
$$

Proof. By Theorem 2.4, we assume that $T_{1}, T_{2}, \ldots, T_{k}$ is a rooted tree partition of $G$, where $k=b(G)$, derived from an optimal burning sequence for $G$. If we take $T$ to be a spanning subtree of $G$ obtained by adding edges between the $T_{i}$ 's that do not induce a cycle in $G$, then $b(T) \leq k=b(G) \leq b(T)$, where the second inequality holds because $T$ is a spanning subgraph of $G$.

A subgraph $H$ of a graph $G$ is called an isometric subgraph if for every pair of nodes $u$ and $v$ in $H$, we have that $d_{H}(u, v)=d_{G}(u, v)$. For example, a subtree of a tree is an isometric subgraph. As another example, if $G$ is a connected graph and $P$ is a shortest path connecting two nodes of $G$, then $P$ is an isometric subgraph of $G$. Let $W_{5}$ be the wheel graph formed by adding a universal node to a 5-cycle. Then, the 5-cycle $C_{5}$ is an isometric subgraph of $W_{5}$, whereas $b\left(C_{5}\right)=3>2=b\left(W_{5}\right)$. Thus, we conclude that the burning number is not monotonic even on the isometric subgraphs of a graph. However, the following theorem shows that the burning number is monotonic on the isometric subgraphs in certain cases.

Theorem 2.6. Let $H$ be an isometric subgraph of a graph $G$ such that, for any node $x \in V(G) \backslash V(H)$, and any positive integer $r$, there exists a node $f_{r}(x) \in V(H)$ for which $N_{r}[x] \cap V(H) \subseteq N_{r}^{H}\left[f_{r}(x)\right]$. Then we have that $b(H) \leq b(G)$.

Proof. It suffices to show that for any optimal burning sequence of $G$ such as $\left(x_{1}, x_{2}, \ldots, x_{k}\right)$, we can assign a burning sequence of length at most $k$ to $H$. Without loss of generality, we may assume that $|V(H)|>k$ (otherwise, $H$ can be burned in at most $|V(H)| \leq k$ steps).

We define the function $f:\left\{x_{1}, x_{2}, \ldots, x_{k}\right\} \rightarrow V(H)$ as follows. For $1 \leq i \leq k$, if $x_{i} \in V(H)$, then we define $f\left(x_{i}\right)=x_{i}$; otherwise, by assumption, there is a node $f_{k-i}\left(x_{i}\right) \in V(H)$ for which $N_{k-i}\left[x_{i}\right] \cap V(H) \subseteq N_{k-i}^{H}\left[f_{k-i}\left(x_{i}\right)\right]$. In this case, we define $f\left(x_{i}\right)=f_{k-i}\left(x_{i}\right)$. Because $H$ is an isometric subgraph of $G$, then for each $x_{i} \in H$, and for every node $v \in N_{k-i}\left[x_{i}\right] \cap V(H)$, we have that $d_{H}\left(x_{i}, v\right)=d_{G}\left(x_{i}, v\right) \leq k-i$. Thus, if $f\left(x_{i}\right)=x_{i}$, then $N_{k-i}\left[x_{i}\right] \cap V(H)=N_{k-i}^{H}\left[x_{i}\right]=N_{k-i}^{H}\left[f\left(x_{i}\right)\right]$. Hence, we derive that

$$
\begin{aligned}
V(H) & =V(G) \cap V(H) \\
& =\left(N_{k-1}\left[x_{1}\right] \cup \ldots \cup N_{0}\left[x_{k}\right]\right) \cap V(H) \\
& =\left(N_{k-1}\left[x_{1}\right] \cap V(H)\right) \cup \ldots \cup\left(N_{0}\left[x_{k}\right] \cap V(H)\right) \\
& \subseteq N_{k-1}^{H}\left[f\left(x_{1}\right)\right] \cup \ldots \cup N_{0}^{H}\left[f\left(x_{k}\right)\right] .
\end{aligned}
$$


Therefore, $\left\{N_{k-i}\left[f\left(x_{i}\right)\right]\right\}_{i=1}^{k}$ forms a covering for the node set of $H$, with $k$ closed neighborhoods. Thus, by Corollary 2.3, we conclude that $b(H) \leq b(G)$.

The following theorem shows that the isometric subtrees of a graph satisfy the conditions in Theorem 2.6.

Theorem 2.7. If $H$ is an isometric subtree of a graph $G$, then $b(H) \leq b(G)$.

Proof. By Theorem 2.6, it suffices to show that for any node $x \in V(G) \backslash V(H)$, and any positive integer $r$, there exists a node $f_{r}(x) \in V(H)$ for which $N_{r}[x] \cap V(H) \subseteq N_{r}^{H}\left[f_{r}(x)\right]$.

Assume that $X_{r}=N_{r}[x] \cap V(H)$. If $X_{r}$ is empty, then we can choose $f_{r}(x)$ to be any node in $H$. If $X_{r}=\{v\}$, then we take $f_{r}(x)=v$, and clearly $N_{r}[x] \cap V(H)=\{v\} \subseteq N_{r}^{H}[v]$. Hence, we assume that $\left|X_{r}\right| \geq 2$. Since $H$ is a tree, then there is a unique path (consisting of the nodes in $H$ only) between every pair of distinct nodes in $X_{r} \subseteq V(H)$. Let $y_{r}$ and $z_{r}$ be two nodes in $X_{r}$ with the maximum distance over all possible pairs of nodes in $X_{r}$, and let $w_{r}$ be a node in $H$ that is of almost equal distance with respect to $y_{r}$ and $z_{r}$. That is, $d\left(w_{r}, y_{r}\right)=d\left(w_{r}, z_{r}\right)$, if $d\left(y_{r}, z_{r}\right)$ is even, and $d\left(w_{r}, z_{r}\right)=d\left(w_{r}, y_{r}\right)+1$ (without of loss of generality) in the case that $d\left(y_{r}, z_{r}\right)$ is odd. We claim that for each $v \in X_{r}, d\left(v, w_{r}\right) \leq r$.

Because, by assumption, $H$ is an isometric subtree of $G$, then the length of the path between $y_{r}$ and $z_{r}$ in $H$ is equal to $d\left(y_{r}, z_{r}\right)$ in $G$. Thus, we have that $d\left(y_{r}, z_{r}\right)=d\left(w_{r}, y_{r}\right)+$ $d\left(w_{r}, z_{r}\right) \leq d\left(x, y_{r}\right)+d\left(x, z_{r}\right)$. To the contrary, we have that $d\left(v, w_{r}\right) \leq d\left(z_{r}, w_{r}\right)$. To show this, we have to consider two possibilities; either $v$ is on the path in $H$ that connects $z_{r}$ to $w_{r}$, or it is not. If the former holds, then obviously, $d\left(v, w_{r}\right) \leq d\left(z_{r}, w_{r}\right)$. If the latter holds, then suppose $u$ is the first node that appears in both paths that connect $v$ and $z_{r}$ to $w_{r}$. If $u=w_{r}$, then we have that

$$
d\left(v, w_{r}\right)+d\left(w_{r}, z_{r}\right)=d\left(v, z_{r}\right) \leq d\left(y_{r}, z_{r}\right)=d\left(y_{r}, w_{r}\right)+d\left(w_{r}, z_{r}\right) .
$$

It implies that $d\left(v, w_{r}\right) \leq d\left(y_{r}, w_{r}\right) \leq d\left(z_{r}, w_{r}\right)$. If $u \neq w_{r}$, then we have that

$$
d\left(v, w_{r}\right)+d\left(w_{r}, y_{r}\right)=d\left(v, y_{r}\right) \leq d\left(z_{r}, y_{r}\right)=d\left(z_{r}, w_{r}\right)+d\left(w_{r}, y_{r}\right) .
$$

Hence, we again conclude that $d\left(v, w_{r}\right) \leq d\left(z_{r}, w_{r}\right)$. Consequently, we have that

$$
\begin{aligned}
d\left(v, w_{r}\right) & \leq d\left(z_{r}, w_{r}\right) \\
& \leq \frac{d\left(y_{r}, z_{r}\right)+1}{2} \\
& \leq \frac{d\left(y_{r}, x\right)+d\left(z_{r}, x\right)+1}{2} \\
& \leq \frac{r+r+1}{2} \\
& =r+\frac{1}{2} .
\end{aligned}
$$

Because $d\left(v, w_{r}\right)$ is an integer, it implies that $d\left(v, w_{r}\right) \leq r$. Therefore, if we define $f_{r}(x)=w_{r}$, then $X_{r} \subseteq N_{r}^{H}\left[f_{r}(x)\right]$. Thus, the proof follows. 
However, the above inequality may fail for nonisometric subtrees. For example, let $H$ be a path of order 5, and form $G$ by adding a universal node (that is, one joined to all others) to $H$. Then $b(H)=3$, but $b(G)=2$.

The following corollary is an immediate consequence of Theorem 2.7.

Corollary 2.8. If $T$ is a tree and $H$ is a subtree of $T$, then we have that $b(H) \leq b(T)$.

The burning number of paths is derived in the following result.

Theorem 2.9. For a path $P_{n}$ on $n$ nodes, we have that $b\left(P_{n}\right)=\left\lceil n^{1 / 2}\right\rceil$.

Proof. Suppose that $\left(x_{1}, x_{2}, \ldots, x_{k}\right)$ is an optimal burning sequence for $P_{n}$. By (2.1), and the fact that for a node $v$ in a path, $\left|N_{i}[v]\right| \leq 2 i+1$, we derive that

$$
\begin{aligned}
\sum_{i=1}^{k}(2(k-i)+1) & =(2(k-1)+2(k-2)+\ldots+2(1))+k \\
& =2\left(\frac{k(k-1)}{2}\right)+k \\
& =k^{2} \geq n .
\end{aligned}
$$

Because $k$ is the minimum number satisfying this inequality, we conclude that $b\left(P_{n}\right) \geq$ $\left\lceil n^{1 / 2}\right\rceil$.

Now, assume that $k=\left\lceil n^{1 / 2}\right\rceil$, and let $P_{n}: v_{1}, v_{2}, \ldots, v_{n}$. Then, for $0 \leq i \leq k-2$, we choose $x_{k-i}=v_{n-i^{2}-i}$. Also, if $n \geq(k-1)^{2}+k$, we take $x_{1}=v_{n-(k-1)^{2}-(k-1)}$; otherwise we take $x_{1}=v_{1}$. Therefore, we can burn $P_{n}$ in exactly $k$ steps by the burning sequence $\left(x_{1}, x_{2}, \ldots, x_{k}\right)$. Hence, $b\left(P_{n}\right) \leq k$. Thus, $b\left(P_{n}\right)=\left\lceil n^{1 / 2}\right\rceil$.

We have the following immediate corollaries.

\section{Corollary 2.10.}

(1) For a cycle $C_{n}$, we have that $b\left(C_{n}\right)=\left\lceil n^{1 / 2}\right\rceil$.

(2) For a graph $G$ of order $n$ with a Hamiltonian (that is, a spanning) path, we have that $b(G) \leq\left\lceil n^{1 / 2}\right\rceil$.

The following theorem gives sharp bounds on the burning number.

Lemma 2.11. For any graph $G$ with radius $r$ and diameter $d$, we have that

$$
\left\lceil(d+1)^{1 / 2}\right\rceil \leq b(G) \leq r+1 .
$$

Proof. Assume that $c$ is a central node of $G$ with eccentricity $r$. Because every node in $G$ is within distance $r$ from $c$, then the fire will spread to all nodes after $r+1$ steps. Hence, $r+1$ is an upper bound for $b(G)$. 
Now, let $P$ be a path connecting two nodes $u$ and $v$ in $G$ with $d(u, v)=d$. Because $P$ is an isometric subtree of $G$, and $|P|=d+1$, by Theorem 2.7 and Theorem 2.9, we conclude that $b(G) \geq b(P)=\left\lceil(d+1)^{1 / 2}\right\rceil$.

The lower bound is achieved by paths. A graph $G$ that is obtained by connecting a disjoint union of paths $\left\{P_{i}\right\}_{i=1}^{t}$, with $t \geq 3$, to a single node $v$ is called a spider graph. The subgraph of $G$ that is induced by each $P_{i} \cup\{v\}$ is called an arm of $G$. The maximum degree of $G$ is the degree of the node $v$ that is at least three. If all the arms of a spider graph with maximum degree $s$ are of the same length $r$, then we denote such a spider graph by $S P(s, r)$. The spider graph $S P(s, 1)$ is called a star graph and is denoted by $K_{1, s}$ mostly in graph theory. The upper bound in Lemma 2.11 is achieved by spider graphs $S P(s, r)$, where $s \geq r$, and also by perfect binary trees (as proven in [7]).

We finish this section by providing some bounds on the burning number in terms of certain domination numbers. A $k$-distance dominating set for $G$ is a subset $D_{k} \subseteq V(G)$ such that for every node $u \in V(G) \backslash D_{k}$, there exists a node $v \in D_{k}$ for which $d(u, v) \leq k$. The number of the nodes in a minimum $k$-distance dominating set of $G$ is denoted by $\gamma_{k}(G)$, and we call it the $k$-distance domination number of $G$. We have the following results on connections between burning and distance domination.

Theorem 2.12. If $G$ is a graph of order at least two and with burning number $k$, then we have that $k \geq \gamma_{k-1}(G)$.

Proof. Assume that $b(G)=k$, for some positive integer $k$, and $\left(x_{1}, x_{2}, \ldots, x_{k}\right)$ is an optimal burning sequence for $G$. Then by (2.1), we know that every node $v$ in $G$ must be within the distance $k-i \leq k-1$ from one of the $x_{i}$ 's. Hence, $D=\left\{x_{1}, x_{2}, \ldots, x_{k}\right\}$ forms a $(k-1)$-distance dominating set for $G$.

We have the following lemma.

Lemma 2.13. For any graph $G$ of order at least two, if $m=\min _{k \geq 1}\left\{\gamma_{k}(G)+k\right\}$, then $\frac{m+1}{2} \leq b(G) \leq m$.

Proof. For simplicity, we denote $\gamma_{k}(G)$ by $\gamma_{k}$. Assume that $m=\min _{k \geq 1}\left\{\gamma_{k}+k\right\}$, and $b(G)=k_{0}$. Then by, Theorem $2.12, b(G)=k_{0} \geq \gamma_{k_{0}-1}$. Hence,

$$
k_{0}+\left(k_{0}-1\right) \geq \gamma_{k_{0}-1}+k_{0}-1 \geq \min _{k \geq 1}\left\{\gamma_{k}+k\right\}=m .
$$

Therefore, $k_{0} \geq \frac{m+1}{2}$.

To the contrary, assume that $D_{k}=\left\{x_{1}, x_{2}, \ldots, x_{\gamma_{k}}\right\}$ is a minimum $k$-distance dominating set for $G$. Then, $\left\{S_{1}, S_{2}, \ldots, S_{\gamma_{k}}\right\}$, with $S_{i}=\left\{v \in V(G): d\left(v, x_{i}\right) \leq k\right\}$, where $1 \leq i \leq \gamma_{k}$, is a covering for the nodes of $G$, which consists of $\gamma_{k}$ subsets each of radius at most $k$. Thus, by Theorem 2.1, we have that $b(G) \leq \gamma_{k}+k$. The result follows because this is true for any $k \geq 1$. Therefore, we have that

$$
\frac{m+1}{2} \leq b(G) \leq m .
$$


We have the following fact about the $k$-distance domination number of graphs.

Theorem 2.14. [12] If $G$ is a connected graph of order $n$, with $n \geq k+1$, then we have that

$$
\gamma_{k}(G) \leq \frac{n}{k+1}
$$

We now provide the following general bound for the burning number of graphs.

Corollary 2.15. If $G$ is a connected graph of order $n$, with $n \geq k+1$, then we have that

$$
b(G) \leq 2\left\lceil n^{1 / 2}\right\rceil-1 .
$$

Proof. By Lemma 2.13 and Theorem 2.14, we derive that for any positive integer $k \leq n-1$,

$$
b(G) \leq \min _{k \geq 1}\left\{\frac{n}{k+1}+k\right\} .
$$

Now, the function $\frac{n}{k+1}+k$ is minimized for $k=\left\lceil n^{1 / 2}\right\rceil-1$, and we note that $k \leq n-1$. Therefore, we have that

$$
\begin{aligned}
b(G) & \leq \min _{k \geq 1}\left\{\frac{n}{k+1}+k\right\} \\
& \leq \frac{n}{\left(\left\lceil n^{1 / 2}\right\rceil-1\right)+1}+\left\lceil n^{1 / 2}\right\rceil-1 \\
& \leq 2\left\lceil n^{1 / 2}\right\rceil-1 .
\end{aligned}
$$

We conjecture that for any connected graph $G$ of order $n, b(G) \leq\left\lceil n^{1 / 2}\right\rceil$, where the bound is achieved by a path on $n$ nodes.

\section{NORDHAUS-GADDUM TYPE RESULTS}

Nordhaus and Gaddum [18] gave bounds on the sum and product of the chromatic number of a graph and its complement in terms of the order of the graph. Analogous relations have been discovered for many other graph parameters; see [2] for a survey. In this section, we present Nordhaus-Gaddum type results for the burning number.

We need first the following simple observation: Let $G$ be a graph of order $n \geq 2$ with maximum degree $\Delta$. If $G$ does not have a universal node, then we have that $b(G) \leq n-\Delta$; otherwise, $b(G)=2$. It follows because we can take a node such as $v$ of degree $\Delta$, and then, by burning $v$ and $V(G) \backslash N[v]$, respectively, we burn all nodes of $G$ in at most $1+|V(G) \backslash N[v]|=n-\Delta$ steps. If $G$ contains a universal node $v$, then by burning $v$ and one of its neighbors, respectively, we can burn $G$ in two steps (note that since $G$ has an edge, we need at least two steps for burning $G$ ). Thus, $b(G)=2$. Also, we need the following theorem from [7]. 
Theorem 3.1. [7] A graph $G$ satisfies $b(G)=2$ if and only if $G$ has order at least 2 and has maximum degree $n-1$ or $n-2$.

We first present some bounds on the sum of the burning numbers of a graph and its complement.

Theorem 3.2. If $G$ is a graph of order $n \geq 2$, then

$$
4 \leq b(G)+b(\bar{G}) \leq n+2 .
$$

Proof. Because the burning number of any graph of order $n \geq 2$ is at least two, then by assumption, we conclude that $b(G)+b(\bar{G}) \geq 2+2=4$. Now, suppose that $b(G)=k$, and $\left(x_{1}, x_{2}, \ldots, x_{k}\right)$ is a burning sequence for $G$. Clearly, $x_{k}$ cannot be adjacent to $x_{i}$, for $1 \leq i \leq k-2$. Therefore, $\Delta(\bar{G}) \geq d_{\bar{G}}\left(x_{k}\right) \geq k-2$. There are two possibilities: either $G$ has an isolated node, or it does not.

If $G$ does not have an isolated node, then $\bar{G}$ does not have a universal node. Thus, by the above observation, $b(\bar{G}) \leq n-\Delta(\bar{G}) \leq n-(k-2)$, and consequently, we have that $b(G)+b(\bar{G}) \leq n+2$.

If $G$ contains an isolated node, then $\bar{G}$ must have a universal node. Therefore, $b(\bar{G})=2$, and clearly, $b(G) \leq n$. Thus, $b(G)+b(\bar{G}) \leq n+2$.

The upper bound in Theorem 3.2 is the best possible, since for the complete graph $K_{n}$ we have that $b\left(\overline{K_{n}}\right)+b\left(K_{n}\right)=n+2$. However, there are cases where the upper bound is strict; for example, $b\left(C_{n}\right)+b\left(\overline{C_{n}}\right)=\left\lceil n^{1 / 2}\right\rceil+3<n+2$. Also, the lower bound in Theorem 3.2 is achieved for the complete graph $K_{2}$, and star graphs $K(1, s)$.

We cite here two useful Nordhaus-Gaddum type results for distance domination.

Theorem 3.3. [2] For any graph $G$ of order $n \geq k+1$ with $k \geq 2$, we have that

$$
\gamma_{k}(G)+\gamma_{k}(\bar{G}) \leq n+1
$$

and

$$
\gamma_{k}(G) \gamma_{k}(\bar{G}) \leq n
$$

Theorem 3.4. [2] If $G$ and $\bar{G}$ are both connected with $n \geq k+1$ nodes for integer $k \geq 2$, then $\gamma_{k}(G)+\gamma_{k}(\bar{G}) \leq \frac{n}{k+1}+1$ and $\gamma_{k}(G) \gamma_{k}(\bar{G}) \leq \frac{n}{k+1}$.

We now have the following result for the product of the burning numbers of a graph and its complement.

Theorem 3.5. For any graph $G$ of order $n$, we have $b(G) b(\bar{G}) \leq 2 n$, and the equality is achieved by complete graphs.

Proof. First, by direct checking, we can see that $b(G) b(\bar{G}) \leq 2 n$, for any graph $G$ of order $n \leq 5$. Now, assume that $G$ is a graph of order $n \geq 6$. By Lemma 2.13, we know that 
$b(G) \leq \gamma_{k}(G)+k$, for $k \geq 1$. Thus, we have that

$$
\begin{aligned}
b(G) b(\bar{G}) & \leq\left(\gamma_{k}(G)+k\right)\left(\gamma_{k}(\bar{G})+k\right) \\
& \leq \gamma_{k}(G) \gamma_{k}(\bar{G})+k \gamma_{k}(G)+k \gamma_{k}(\bar{G})+k^{2} .
\end{aligned}
$$

If $G$ and $\bar{G}$ are both connected and $n \geq k+1$ where $k \geq 2$, then using Theorem 3.4, we have that

$$
\begin{aligned}
b(G) b(\bar{G}) & \leq \gamma_{k}(G) \gamma_{k}(\bar{G})+k \gamma_{k}(G)+k \gamma_{k}(\bar{G})+k^{2} \\
& \leq \frac{n}{k+1}+k\left(\frac{n}{k+1}+1\right)+k^{2} \\
& =n+k+k^{2} .
\end{aligned}
$$

By taking $k=2$, the above inequality implies that $b(G) b(\bar{G}) \leq n+6$.

If $G$ is connected while $\bar{G}$ is disconnected, then, either $\bar{G}$ has a component with at most two nodes, or every component of $\bar{G}$ has at least three nodes. If $\bar{G}$ has an isolated node, then $G$ must have a universal node. If $\bar{G}$ has a component with exactly two nodes, then $G$ must contain two nodes such as $u$ and $v$ (corresponding to the component of $\bar{G}$ with exactly two nodes), such that $G=N[u] \cup\{v\}$. Thus, if $\bar{G}$ has a component with at most two nodes, then by Theorem $3.1, b(G)=2$, and obviously $b(\bar{G}) \leq n$. Hence, in this case, $b(G) b(\bar{G}) \leq 2 n$.

Now, suppose that $G_{1}, G_{2}, \ldots, G_{t}$ are the components of $\bar{G}$ with $n_{1}, n_{2}, \ldots, n_{t}$ nodes, respectively, where each $n_{i} \geq 3$. By Theorem 2.14 , and taking $k=2$, we know that

$$
\begin{aligned}
\gamma_{2}(\bar{G}) & =\gamma_{2}\left(G_{1}\right)+\gamma_{2}\left(G_{2}\right)+\cdots+\gamma_{2}\left(G_{t}\right) \\
& \leq \frac{n_{1}}{3}+\frac{n_{2}}{3}+\cdots+\frac{n_{t}}{3} \\
& =\frac{n}{3} .
\end{aligned}
$$

Also, note that $b(G) \leq 3$, since we can easily see that in such a case the radius of $G$ is at most 2. Therefore, by Lemma 2.13 and Theorem 2.14 , for $k=2$, we have that

$$
\begin{aligned}
b(G) b(\bar{G}) & \leq 3\left(\gamma_{2}(\bar{G})+2\right) \\
& \leq 3\left(\frac{n}{3}+2\right) \\
& =n+6,
\end{aligned}
$$

and the proof follows.

Corollary 3.6. If graphs $G$ and $\bar{G}$ are connected graphs of order $n \geq 6$, then $b(G)+$ $b(\bar{G}) \leq 3\left\lceil n^{1 / 2}\right\rceil-1$, and $b(G) b(\bar{G}) \leq n+6$.

Proof. First, by Lemma 2.12, we have that

$$
\begin{aligned}
b(G)+b(\bar{G}) & \leq\left(\gamma_{k}(G)+k\right)+\left(\gamma_{k}(\bar{G})+k\right) \\
& =\gamma_{k}(G)+\gamma_{k}(\bar{G})+2 k .
\end{aligned}
$$


By applying Theorem 3.4 with $k=\left\lceil n^{1 / 2}\right\rceil-1$, we conclude that

$$
\begin{aligned}
b(G)+b(\bar{G}) & \leq \frac{n}{k+1}+1+2 k \\
& \leq 3\left\lceil n^{1 / 2}\right\rceil-1 .
\end{aligned}
$$

Finally, $b(G) b(\bar{G}) \leq n+6$ was shown in the proof of Theorem 3.5.

We conjecture that if $G$ and $\bar{G}$ are both connected graphs of order $n$, then we have that $b(G) b(\bar{G}) \leq n+4$. It is straightforward to see that the bound in the conjecture is tight for the cycle $C_{5}$.

\section{BURNING IN THE ILT MODEL}

The Iterated Local Transitivity (ILT) model was introduced in [6], and simulates online social networks (or OSNs). The central idea behind the ILT model is what sociologists call transitivity: if $u$ is a friend of $v$, and $v$ is a friend of $w$, then $u$ is a friend of $w$. In its simplest form, transitivity gives rise to the notion of cloning, where $u$ is joined to all of the neighbors of $v$. In the ILT model, given some initial graph as a starting point, nodes that clone each node are repeatedly added over time so that the new nodes form an independent set. The only parameter of the model is the initial graph $G_{0}$, which is any fixed finite connected graph. Assume that for a fixed $t \geq 0$, the graph $G_{t}$ has been constructed. To form $G_{t+1}$, for each node $x \in V\left(G_{t}\right)$, add its clone $x^{\prime}$, such that $x^{\prime}$ is joined to $x$ and all of its neighbors at time $t$. Note that the set of new nodes at time $t+1$ form an independent set of cardinality $\left|V\left(G_{t}\right)\right|$.

The ILT model shares many properties with OSNs, such as low average distance, highclustering coefficient densification, and bad spectral expansion; see [6]. The ILT model has also been studied from the viewpoint of competitive diffusion, which is one model of the spread of influence; see [20].

We have the following theorem about the burning number of graphs obtained based on ILT model. Even though the graphs generated by the ILT model grow exponentially in order with $t$, we see that the burning number of such networks remains constant.

Theorem 4.1. Let $G_{t}$ be the graph generated at time $t \geq 1$ based on the ILT model with initial graph $G_{0}$. If $G_{0}$ has an optimal burning sequence $\left(x_{1}, x_{2}, \ldots, x_{k}\right)$ in which $x_{k}$ has a neighbor that is burned in the $(k-1)$ th step, then $b\left(G_{t}\right)=b\left(G_{0}\right)$. Otherwise, $b\left(G_{t}\right)=b\left(G_{0}\right)+1$.

Proof. First, assume that $\left(x_{1}, x_{2}, \ldots, x_{k}\right)$ is an optimal burning sequence for $G_{0}$. Since every node $x^{\prime} \in V\left(G_{t}\right) \backslash V\left(G_{0}\right)$, with $t \geq 1$, is adjacent to a node in $G_{0}$, we have that $\left(x_{1}, x_{2}, \ldots, x_{k}\right)$ is also a burning sequence for the subgraph of $G_{t}$ induced by $V\left(G_{t}\right) \backslash$ $\left(N^{G_{t}}\left[x_{k}\right] \backslash N^{G_{0}}\left[x_{k}\right]\right)$. Thus, $b\left(G_{t}\right) \leq b\left(G_{0}\right)+1$. With a similar argument, we conclude that $b\left(G_{t}\right) \leq b\left(G_{t-1}\right)+1$.

However, we can easily see that $G_{t-1}$ is an isometric subgraph of $G_{t}$, for any $t \geq 1$. Also, for any $x \in V\left(G_{t-1}\right)$ and its clone $x^{\prime} \in V\left(G_{t}\right)$, we have that $N^{G_{t}}[x]=N^{G_{t}}\left[x^{\prime}\right]$. Thus, for any $r \geq 1, N_{r}\left[x^{\prime}\right] \cap V\left(G_{t-1}\right)=N_{r}^{G_{t-1}}[x]$. Therefore, by Theorem 2.6, $b\left(G_{t}\right) \geq b\left(G_{t-1}\right)$. Hence, by induction we conclude that $b\left(G_{t}\right) \geq b\left(G_{0}\right)$, for any $t \geq 1$, and therefore, we 
have that either $b\left(G_{t}\right)=b\left(G_{0}\right)$, or $b\left(G_{t}\right)=b\left(G_{0}\right)+1$. We now characterize where $b\left(G_{t}\right)$ equals $b\left(G_{0}\right)$ or $b\left(G_{t}\right)=b\left(G_{0}\right)+1$ as follows.

Let $\left(x_{1}, x_{2}, \ldots, x_{k}\right)$ be an optimal burning sequence for $G_{t}$. By the following algorithm, we find a burning sequence $\left(y_{1}, y_{2}, \ldots, y_{k}\right)$ for $G_{t}$ wherein at least all the first $k-1$ fire sources are in $G_{t-1}$. Note that, by this argument, either $b\left(G_{t-1}\right)=k$ or $b\left(G_{t-1}\right)=k-1$.

Step 1. If $x_{1} \in V\left(G_{t-1}\right)$, then we take $y_{1}=x_{1}$. Otherwise, we set $y_{1}=x$, where $x$ is a node in $V\left(G_{t-1}\right)$.

Go to Step 2.

Step 2. For each $2 \leq i \leq k-1$, we do the following steps:

Step 2.1. If $x_{i} \in V\left(G_{t-1}\right)$ and $x_{i}$ is not burned in step $i-1$ by burning $y_{1}, y_{2}, \ldots, y_{i-1}$, then we take $y_{i}=x_{i}$.

Step 2.2. If $x_{i} \in V\left(G_{t-1}\right)$ and $x_{i}$ is burned in step $i-1$ by burning $y_{1}, y_{2}, \ldots, y_{i-1}$, then we set $y_{i}=x$, where $x$ is a node in $V\left(G_{t-1}\right)$ that is not burned in step $i-1$. We are sure that such a node $x$ exists, because $b\left(G_{t-1}\right) \geq k-1$.

Step 2.3. If $x_{i}$ is the clone of $x_{i}^{\prime} \in V\left(G_{t-1}\right)$, and $x_{i}^{\prime}$ is not burned in step $i-1$ by burning $y_{1}, y_{2}, \ldots, y_{i-1}$, then we take $y_{i}=x_{i}^{\prime}$.

Step 2.4. If $x_{i}$ is the clone of $x_{i}^{\prime} \in V\left(G_{t-1}\right)$, and $x_{i}^{\prime}$ is burned in step $i-1$ by burning $y_{1}, y_{2}, \ldots, y_{i-1}$, then we set $y_{i}=x$, where $x$ is a node in $V\left(G_{t-1}\right)$ that is not burned in step $i-1$. We are sure that such a node $x$ exists, because $b\left(G_{t-1}\right) \geq k-1$.

Go to Step 3.

Step 3. We perform the following steps, and we return the sequence $\left(y_{1}, y_{2}, \ldots, y_{k}\right)$ as a burning sequence for $G_{t}$.

Step 3.1. If $x_{k} \in V\left(G_{t-1}\right)$, and $x_{k}$ is not burned in step $k-1$ by burning $y_{1}, y_{2}, \ldots, y_{k-1}$, then we take $y_{k}=x_{k}$.

Step 3.2. If $x_{k} \in V\left(G_{t-1}\right)$, and $x_{k}$ is burned in step $k-1$ by burning $y_{1}, y_{2}, \ldots, y_{k-1}$, then we take $y_{k}=x$, where $x$ is a node in $V\left(G_{t-1}\right)$ that is not burned in step $k-1$, if such a node $x$ is available; Otherwise, we choose $x$ to be a node in $V\left(G_{t}\right)$ that is not burned in step $t-1$.

Step 3.3. If $x_{k}$ is the clone of $x_{k}^{\prime} \in V\left(G_{t-1}\right)$, and $x_{k}^{\prime}$ is not burned in step $k-1$ by burning $y_{1}, y_{2}, \ldots, y_{k-1}$, then we take $y_{k}=x_{k}^{\prime}$.

Step 3.4. If $x_{k}$ is the clone of $x_{k}^{\prime} \in V\left(G_{t-1}\right)$, and $x_{k}^{\prime}$ is burned in step $k-1$ by burning $y_{1}, y_{2}, \ldots, y_{k-1}$, then we set $y_{k}=x$, where $x$ is a node in $V\left(G_{t-1}\right)$ that is not burned in step $k-1$, if such a node $x$ is available; Otherwise, we choose $x$ to be a node in $V\left(G_{t}\right)$ that is not burned in step $t-1$.

The sequence $\left(y_{1}, y_{2}, \ldots, y_{k}\right)$ obtained by this algorithm is a burning sequence for $G_{t}$. Namely, by burning the nodes in the sequence $\left(y_{1}, y_{2}, \ldots, y_{k}\right)$, each node $x_{i}$ or its clone $x_{i}^{\prime}$ is burning at stage $i$, for $1 \leq i \leq k$. Therefore, for $1 \leq i \leq k, N_{k-i}^{G_{t}}\left[x_{i}\right]$ or $N_{k-i}^{G_{t}}\left[x_{i}^{\prime}\right]$ is burned by the end of the $k$ th step. Since for $1 \leq i \leq k, N^{G_{t}}\left[x_{i}\right]=N^{G_{t}}\left[x_{i}^{\prime}\right]$, then we have 
$N_{k-i}^{G_{t}}\left[x_{i}\right]=N_{k-i}^{G_{t}}\left[x_{i}^{\prime}\right]$. Hence, it implies that

$$
\begin{aligned}
V\left(G_{t}\right) & \supseteq N_{k-1}^{G_{t}}\left[y_{1}\right] \cup N_{k-2}^{G_{t}}\left[y_{2}\right] \cup \ldots \cup N_{0}^{G_{t}}\left[y_{k}\right] \\
& \supseteq N_{k-1}^{G_{t}}\left[x_{1}\right] \cup N_{k-2}^{G_{t}}\left[x_{2}\right] \cup \ldots \cup N_{0}^{G_{t}}\left[x_{k}\right] \\
& =V\left(G_{t}\right) .
\end{aligned}
$$

Thus, by (2.1), we conclude that $\left(y_{1}, y_{2}, \ldots, y_{k}\right)$ is a burning sequence for $G_{t}$.

Suppose that for every optimal burning sequence $\left(x_{1}, x_{2}, \ldots, x_{k}\right)$ of $G_{0}$ all the neighbors of $x_{k}$ are burned in the $k$ th step. We claim that $b\left(G_{1}\right)=b\left(G_{0}\right)+1$. Do not assume that, $b\left(G_{1}\right)=b\left(G_{0}\right)$. Let $\left(y_{1}, y_{2}, \ldots, y_{k}\right)$ be an optimal burning sequence for $G_{1}$ that is obtained from an optimal burning sequence $\left(z_{1}, z_{2}, \ldots, z_{k}\right)$ for $G_{1}$ by the preceding algorithm. Hence, $\left\{y_{1}, \ldots, y_{k}\right\} \subseteq G_{0}$. Otherwise, it implies that $b\left(G_{0}\right)=k-1$, which is a contradiction. But, then to burn $y_{k}^{\prime} \in V\left(G_{1}\right)$ (the clone of $y_{k}$ ) by the end of the $k$ th step, one of the nodes in the neighborhood of $y_{k}$ must be burned in an earlier stage, which is a contradiction with the assumption. Therefore, in this case $b\left(G_{1}\right)=b\left(G_{0}\right)$ is impossible, and hence, $b\left(G_{1}\right)=b\left(G_{0}\right)+1$.

Conversely, suppose that $b\left(G_{1}\right)=b\left(G_{0}\right)+1$, and $\left(x_{1}, x_{2}, \ldots, x_{k}\right)$ is an optimal burning sequence for $G_{0}$. If $x_{k}$ has a neighbor that is burned at stage $k-1$, then $x_{k}^{\prime}$ is also burned at stage $k$. Therefore, $\left(x_{1}, x_{2}, \ldots, x_{k}\right)$ is also a burning sequence for $G_{1}$, and we have that $b\left(G_{1}\right)=b\left(G_{0}\right)$, which is a contradiction. Thus, $b\left(G_{1}\right)=b\left(G_{0}\right)+1$, if and only if for every optimal burning sequence of $G_{0}$, say $\left(x_{1}, x_{2}, \ldots, x_{k}\right)$, all the neighbors of $x_{k}$ are burned in stage $k$. By induction, we can conclude that $b\left(G_{t}\right)=b\left(G_{0}\right)+1$ if and only if for every optimal burning sequence of $G_{0}$, say $\left(x_{1}, x_{2}, \ldots, x_{k}\right)$, all the neighbors of $x_{k}$ are burned in stage $k$. Since starting from any graph $G_{0}$, for any $t \geq 1$, either $b\left(G_{t}\right)=b\left(G_{0}\right)$, or $b\left(G_{t}\right)=b\left(G_{0}\right)+1$, we conclude that $b\left(G_{t}\right)=b\left(G_{0}\right)$ if and only if for every optimal burning sequence of $G_{0}$, say $\left(x_{1}, x_{2}, \ldots, x_{k}\right)$ one of the neighbors of $x_{k}$ is burned at stage $k-1$.

We finish this section with an example that illustrates Theorem 4.1. Let $P_{n}$ be a path on $n$ nodes such that $\left\lceil n^{1 / 2}\right\rceil=k$, for some positive integer $k$. Then by Theorem 2.9, we know that $b\left(P_{n}\right)=k$. Moreover, if $\left(x_{1}, x_{2}, \ldots, x_{k}\right)$ is an optimal burning sequence for $P_{n}$, then burning $P_{n}$ is equivalent to decomposing $P_{n}$ into paths of orders at most $1,3, \ldots, 2 k-1$, in which each path is a rooted path of radius at most $k-i$ and with root $x_{i}$, for some $1 \leq i \leq k$. Thus, we can easily see that $x_{k}$ is the path of order 1 in such a decomposition for $P_{n}$ in terms of neighborhoods of $x_{i}$ 's. There are two possibilities for $n$; either $n=k^{2}$, or $n \neq k^{2}$.

If $n=k^{2}$, then it implies that the order of each path in decomposing $P_{n}$ is exactly equal to $2(k-i)+1$, for some $1 \leq i \leq k$. Therefore, the end points of such paths are burned in the $k$ th steps. Hence, both neighbors or the only neighbor of $x_{k}$ must burn in the $k$ th step, depending on the position of $x_{k}$ in $P_{n}$. Thus, by Theorem 4.1, if $G_{0}=P_{n}$ in the ILT model, then we have that $b\left(G_{t}\right)=b\left(P_{n}\right)+1=k+1$, for $t \geq 1$.

Otherwise, if $n \neq k^{2}$, then, there is at least one $i$ for which the rooted path with root $x_{i}$ is of order less than $2(k-i)+1$. That is, one of the end points of this path called $x$ is not burned at the $k$ th step. If in decomposing $P_{n}$, we choose $x_{k}$ to be the neighbor of $x$, then we have a burning sequence for $P_{n}$ such that at least one of the neighbors of $x_{k}$ is not burned at step $k$. Therefore, by Theorem 4.1 , if $G_{0}=P_{n}$ in the ILT model, then we have that $b\left(G_{t}\right)=b\left(P_{n}\right)=k$. 


\section{CONCLUSIONS AND FUTURE WORK}

We introduced a new graph parameter, the burning number of a graph, written $b(G)$. The burning number measures how rapidly social contagion spreads in a given graph. We gave a characterization of the burning number in terms of decompositions into trees and gave bounds on the burning number that allow us to compute it for a variety of graphs. We showed the strong connection between the burning number and the distance domination, which we use for finding bounds on the burning number, as well as proving Nordhaus-Gaddum-type results on the burning number of a graph and its complement. We determined the burning number in the Iterated Local Transitive model for social networks.

Several problems remain with regard to the burning number. We conjecture that, for a connected graph $G$ of order $n, b(G) \leq\left\lceil n^{1 / 2}\right\rceil$. Determining the burning number remains open for many classes of graphs, including trees and disconnected graphs. It remains open to consider the burning number in real-world social networks such as Facebook or LinkedIn. As Theorem 4.1 suggests, the burning number of online social networks is likely of constant order as the network grows over time. We remark that the burning number generalizes naturally to directed graphs; one interesting direction is to determine the burning number on Kleinberg's small world model [15], which adds random directed edges to the Cartesian grid.

A simple variation, which leads to complex dynamics, is to change the rules for nodes to burn. As in graph bootstrap percolation [3], the rules could be varied so nodes burn only if they are adjacent to at least $r$ burned neighbors, where $r>1$. We plan on studying this variation in future work.

\section{FUNDING}

Supported by grants from NSERC

\section{REFERENCES}

[1] N. Alon, P. Prałat, and N. Wormald. "Cleaning Regular Graphs with Brushes." SIAM Journal on Discrete Mathematics 23 (2008), 233-250.

[2] M. Aouchiche, and P. Hansen. "A Survey of Nordhaus-Gaddum Type Relations." Discrete Applied Mathematics 161 (2013), 466-546.

[3] J. Balogh, B. Bollobás, and R. Morris. "Graph Bootstrap Percolation.” Random Structures and Algorithms 41 (2012), 413-440.

[4] S. Banerjee, A. Das, A. Gopalan, and S. Shakkottai. "Epidemic Spreading with External Agents." IEEE Transactions on Information Theory (July 2014).

[5] A. Barghi, and P. Winkler. "Firefighting on a Random Geometric Graph." Random Structures and Algorithms 46 (2015), 466-477.

[6] A. Bonato, N. Hadi, P. Horn, P. Prałat, and C. Wang. "Models of On-line Social Networks." Internet Mathematics 6 (2011), 285-313.

[7] A. Bonato, J. Janssen, and E. Roshanbin. "Burning a graph is hard." Preprint (2015).

[8] A. Bonato, and R. J. Nowakowski. The Game of Cops and Robbers on Graphs. Providence, Rhode Island: American Mathematical Society, 2011.

[9] P. Domingos, and M. Richardson. "Mining the Network Value of Customers." In Proceedings of the 7th International Conference on Knowledge Discovery and Data Mining (KDD), 2001.

[10] S. Finbow, A. King, G. MacGillivray, and R. Rizzi. "The Firefighter Problem for Graphs of Maximum Degree Three.” Discrete Mathematics 307 (2007), 2094-2105.

[11] S. Finbow, and G. MacGillivray. "The Firefighter Problem: A Survey of Results, Directions and Questions.” Australasian Journal of Combinatorics 43 (2009), 57-77. 
[12] T. W. Haynes, S. T. Hedetniemi, and P. J. Slater. Fundamentals of Domination in Graphs. New York, NY: Marcel Dekker, 1998.

[13] D. Kempe, J. Kleinberg, É. Tardos. "Maximizing the Spread of Influence Through a Social Network." In Proceedings of the 9th International Conference on Knowledge Discovery and Data Mining (KDD), 2003.

[14] D. Kempe, J. Kleinberg, and E. Tardos. "Influential Nodes in a Diffusion Model for Social Networks." In Proceedings 32nd International Colloquium on Automata, Languages and Programming (ICALP), 2005.

[15] J. Kleinberg. "The Small-World Phenomenon: An Algorithmic Perspective." In Proceedings of the 32nd ACM Symp. Theory of Computing. New York, NY: ACM, 2000.

[16] A. D. I. Kramer, J. E. Guillory, and J. T. Hancock. "Experimental Evidence of Massive-Scale Emotional Contagion Through Social Networks, Proceedings of the National Academy of Sciences 111 (2014), 8788-8790.

[17] E. Mossel, and S. Roch. "On the Submodularity of Influence in Social Networks." In: Proceedings of 39th Annual ACM Symposium on Theory of Computing(STOC), 2007.

[18] E. A. Nordhaus, and J. Gaddum. "On Complementary Graphs." Amer. Math. Monthly 63 (1956), $175-177$.

[19] M. Richardson, and P. Domingos. "Mining Knowledge-Sharing Sites for Viral Marketing." In Proceedings of the 8th International Conference on Knowledge Discovery and Data Mining (KDD), 2002.

[20] L. Small, and O. Mason. "Information Diffusion on the Iterated Local Transitivity Model of Online Social Networks." Discrete Applied Mathematics 161 (2013), 1338-1344.

[21] D. B. West. Introduction to Graph Theory, 2nd edition, Upper Saddle River, NJ: Prentice Hall, 2001. 